\title{
VASSILIEV HOMOTOPY STRING LINK INVARIANTS
}

\author{
DROR BAR-NATAN
}

Appeared in Journal of Knot Theory and its Ramifications 4-1 (1995) 13-32.

\begin{abstract}
We investigate Vassiliev homotopy invariants of string links, and find that in this particular case, most of the questions left unanswered in [3] can be answered affirmatively. In particular, Vassiliev invariants classify string links up to homotopy, and all Vassiliev homotopy string link invariants come from marked surfaces as in [3], using the same construction that in the case of knots gives the HOMFLY and Kauffman polynomials. Alongside, the Milnor $\mu$ invariants of string links are shown to be Vassiliev invariants, and it is re-proven, by elementary means, that Vassiliev invariants classify braids.
\end{abstract}

\section{Contents}

1. Introduction 1

2. Vassiliev invariants of string links 3

2.1. A brief review of [3] 3

2.2. Vassiliev invariants of string links 4

3. Vassiliev homotopy string link invariants 6

4. Vassiliev invariants classify braids 9

4.1. Braids $\quad 9$

4.2. Braids with double points 11

5. On the Milnor invariants 13

5.1. Vassiliev invariants classify string links up to homotopy 13

5.2. The Milnor $\mu$ invariants 14

5.3. Some more Milnor $\mu$ invariants $\quad 15$

6. Odds and ends 16

6.1. Some questions 16

6.2. Acknowledgement 16

$\begin{array}{ll}\text { References } & 16\end{array}$

\section{INTRODUCTION}

In $[22,23]$, Vassiliev introduced a particularly simple class $\mathcal{V}$ of knot invariants, which later where shown by the author $[1,2,3]$, by Birman and Lin [5, 14], and by Gusarov [8] to be at least as strong as all the known knot polynomials. Vassiliev has noticed that to every Vassiliev invariant corresponds a linear functional on some combinatorially defined

Date: This edition: February 17, 2015; First edition: February 1993.

This work was supported by NSF grant DMS-92-03382.

This paper is available electronically at http://www.ma.huji.ac.il/ ${ }^{d}$ rorbn. 
vector space $\mathcal{A}$, and later, Kontsevich (see [3]) has proven that (up tp a minor additional condition), every linear functional on $\mathcal{A}$ comes from a Vassiliev invariant. Furthermore, Kontsevich found a simple way to associate a linear functional on $\mathcal{A}$ to every compact twodimensional surface marked in a certain way. Building on this work of Kontsevich, in [3] the author was able to show that the invariants corresponding to such marked two-dimensional surfaces are exactly the HOMFLY and Kauffman polynomials, together with all of their cablings. As of now, the following two questions are still unanswered:

Q1 How strong are Vassiliev invariants? How close do they come to separating knots?

Q2 Do all Vassiliev invariants come from marked surfaces?

The purpose of this paper is to answer these two questions affirmatively for the case of string links considered up to homotopy. For completeness, let us recall here the definitions of string links and of homotopies between string links:

Definition 1.1. (Habegger-Lin [9]) Let $k$ be a positive integer, let $I$ be the unit interval $[0,1]$, let $D$ be the unit disk, and let $\left\{p_{i}\right\}_{i=1}^{k}$ be some fixed choice of $k$ points in $D$. A $k$-component string link (or simply a string link) in $D \times I$ is a smooth embedding

$$
\sigma: \coprod_{i=1}^{k} I_{i} \rightarrow D \times I
$$

of $k$ disjoint copies $I_{1}, \ldots, I_{k}$ of the unit interval $\mathrm{I}$ in the cylinder $D \times I$, such that $\left.\sigma\right|_{I_{i}}(\epsilon)=$ $p_{i} \times \epsilon$ for $\epsilon=0,1$. Two string links $\sigma_{0}$ and $\sigma_{1}$ are considered equivalent if there exists a one parameter family $\sigma_{t}$ of string links interpolating between them; in other words, if one can get from one to the other via a sequence of Reidemeister moves preserving the endpoints $\left\{p_{i}\right\} \times\{0,1\}$ (see e.g. [11]). Two string links $\sigma_{0}$ and $\sigma_{1}$ are called homotopic if there exists an end-point preserving homotopy $\sigma_{t}$ between the maps $\sigma_{0}$ and $\sigma_{1}$, along which the images of the $I_{i}$ 's are always disjoint (however, for any given $t$ and $i$ the map $\left.\sigma_{t}\right|_{I_{i}}$ is not required to be injective, namely, strands of the string link are allowed to self-intersect freely during a homotopy). Normally we will associate a color $v_{i}$ to each of the points $p_{i}$, and hence to each of the strands of a string link $\sigma$. See figure 1 .
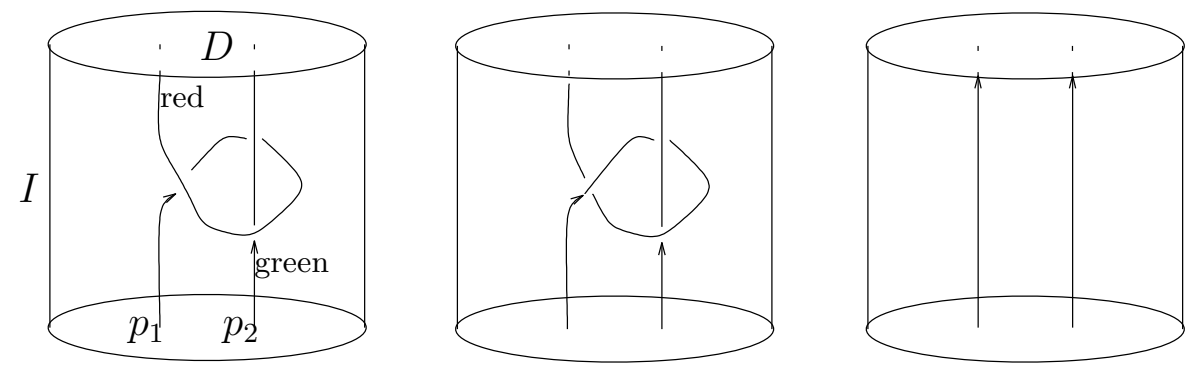

Figure 1. Three inequivalent 2-component string links. The first two are homotopic to each other, while the third is not homotopic to any of the first two.

While answering questions Q1 and Q2 for homotopy string links, we will also find that:

- Vassiliev invariants separate braids. 
- The Milnor $\mu$ invariants (see e.g. Milnor [17, 18] and Habegger-Lin [9]) are Vassiliev invariants (see also Lin [15]), coming from the same single uniform construction as the HOMFLY and Kauffman polynomials.

\section{VASSILIEV INVARIANTS OF STRING LINKS}

2.1. A brief review of [3]. Let us briefly recall the main results of [3] $]^{1}$. In [3], the following vector spaces (over some ground field $\mathbb{F}$ of characteristic 0 ) and linear maps were considered at length:

$$
\begin{aligned}
& \mathcal{K} \underset{1-1 ?}{\stackrel{V^{*}}{\longrightarrow}} \overline{\mathcal{A}} \supset \mathcal{A} \underset{\bar{\sigma}}{\stackrel{\bar{\chi}}{\longrightarrow}} \mathcal{B} \underset{1-1 ?}{\stackrel{\Phi}{\longrightarrow}} \mathcal{M} \\
& \mathcal{K}^{*} \supset \mathcal{V} \underset{V}{\stackrel{W_{m}}{\longrightarrow}} \mathcal{W} \underset{\leftarrow}{\subset} \mathcal{A}^{*} \underset{\bar{\sigma}^{*}}{\stackrel{\bar{\chi}^{*}}{\longrightarrow}} \mathcal{B}^{*} \underset{\text { onto? }}{\stackrel{\Phi^{*}}{\stackrel{1}{*}}} \mathcal{M}^{*}
\end{aligned}
$$

In the above two diagrams,

- $\mathcal{K}$ is the vector space freely generated by all oriented knots in the oriented Euclidean space $\mathbb{R}^{3}$, and $\mathcal{K}^{*}$ is its dual, the space of all $\mathbb{F}$-valued knot invariants.

- $\mathcal{V}$ is a certain subspace of $\mathcal{K}^{*}$, containing the so-called "Vassiliev knot invariants". $\mathcal{V}$ is a filtered vector space, with the type $m$ subspace $\mathcal{F}_{m} \mathcal{V}$ being the space of all invariants vanishing on knots having more than $m$ self-intersections; recall that any knot invariant $V$ can be extended to knots with self-intersections via the formula

$$
V(\nearrow)=V(\nearrow)-V(\nearrow) \text {. }
$$

This formula can be thought of as analogous to differentiation, and Vassiliev invariants of type $m$ can be thought of as invariants whose $(m+1)$ st derivative vanishes.

- $\mathcal{A}$ is the quotient space of the graded space $\mathcal{D}^{l}$ of all linear diagrams by the subspace spanned by all $S T U$ relations. A linear diagram is a diagram made of a single directed full line, some dashed arcs some of whose ends are on the full line, and some oriented trivalent vertices in which three dashed lines meet. The STU relation is the relation

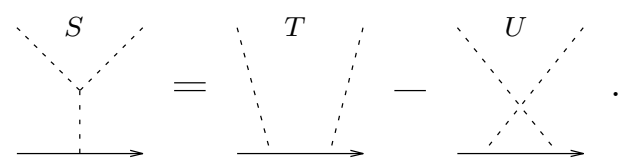

One can show that the following two relations also hold in $\mathcal{A}$ :

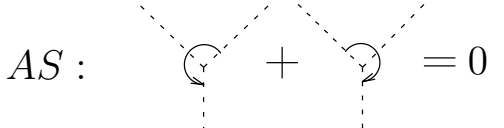

$I H X:$

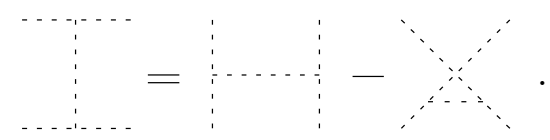

- $\overline{\mathcal{A}}$ is the graded completion of $\mathcal{A}, \mathcal{A}^{*}$ is the graded dual of $\mathcal{A}$, and $\mathcal{W}$ is a certain subspace of $\mathcal{A}^{*}$, from which $\mathcal{A}^{*}$ can be easily reconstructed. There is a naturally defined projection $\mathcal{A}^{*} \rightarrow \mathcal{W}$.

- The map $W_{m}$ is the analogue of computing the (constant) $m$ th derivative of a Vassiliev invariant of type $m$, and is defined only on $\mathcal{F}_{m} \mathcal{V} . V$ is the much harder operation of "integration", and presently it can only be defined via transcendental methods (via Chern-Simons theory or using the Knizhnik-Zamolodchikov connection). $V^{*}$ was not considered in [3], but can easily be defined as the adjoint of $V$. A pivotal question in

\footnotetext{
${ }^{1}$ It is a good idea to have a copy of [3] around while reading this paper.
} 
this context is whether the map $V^{*}$ is one-to-one, or, equivalently, whether Vassiliev invariants are fine enough to separate all knots.

- $\mathcal{A}$ is a Hopf algebra: its (commutative!) product is defined by juxtaposition, while the (commutative) product on $\mathcal{A}^{*}$ is inherited from the obvious product on knot invariants in $\mathcal{V}$.

- The space $\mathcal{B}$ is the quotient space of the graded space $\mathcal{C}$ of all "Chinese characters" by the subspace spanned by all $A S$ and $I H X$ relations. A Chinese character is a diagram made of the same ingredients as the diagrams in $\mathcal{D}^{l}$, only that the directed full line is replaced by some number of univalent vertices. $\mathcal{A}$ and $\mathcal{B}$ are isomorphic by an analogue of the Poincare-Birkhoff-Witt (PBW) theorem.

- $\mathcal{M}$ is the vector space spanned by all marked surfaces. A marked surface is a compact two dimensional smooth surface with a choice of finitely many tangents to its boundary, regarded up to a diffeomorphism. The map $\Phi$ is the composition of two maps: the marking map $\mu$ defined by the relation

and the thickening map defined by the figure

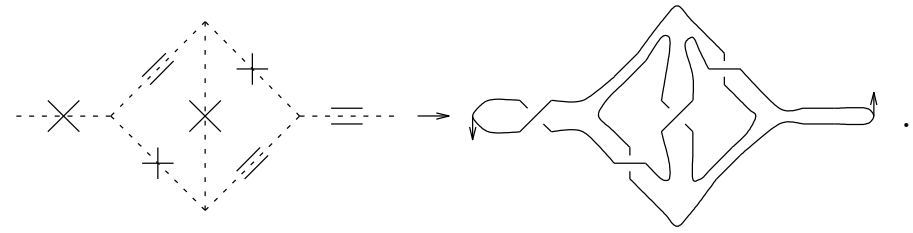

- The image of $\mathcal{M}^{*}$ in $\mathcal{K}^{*}$ is the space of knot invariants coming from the HOMFLY [10] and Kauffman [12] polynomials and all of their cablings. A second pivotal question in this context is whether the map $\Phi$ is one-to-one. Indeed, $\Phi$ is injective iff the class of Vassiliev invariants is precisely as strong as the HOMFLY and Kauffman polynomials and all of their cablings.

- The spaces above are all graded (or filtered) in compatible ways. For more specific information about their gradings, consult e.g. [3].

2.2. Vassiliev invariants of string links. Let us fix the number $k$ of strands in a string links, as well as a list of colors $\Upsilon=\{$ red, green, ..., cyan $\}$ for these strands. The reader should have little difficulty convincing herself that in the case of string links, diagram (1) is replaced by

$$
\begin{aligned}
& \mathcal{K}^{s l} \underset{1-1 ?}{\stackrel{V^{s l *}}{\longrightarrow}} \overline{\mathcal{A}}^{s l} \supset \mathcal{A}^{s l} \underset{\bar{\sigma}^{s l}}{\stackrel{\bar{x}^{s l}}{\longrightarrow}} \mathcal{B}^{s l} \underset{1-1 ?}{\stackrel{\Phi^{s l}}{\longrightarrow}} \mathcal{M}^{s l}
\end{aligned}
$$

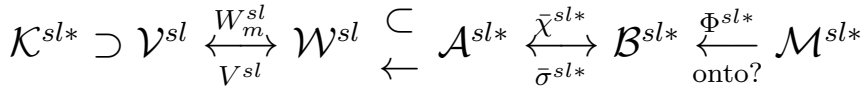

The main points to notice are:

- $\mathcal{K}^{s l}$ is now spanned by all string links.

- Vassiliev invariants are defined in exactly the same way, using (2). $\mathcal{V}^{s l}$ is the space of Vassiliev invariants. It is a filtered space, and its type $m$ subspace is denoted by $\mathcal{F}_{m} \mathcal{V}^{s l}$. 
- $\mathcal{A}^{s l}$ is the space of diagrams like

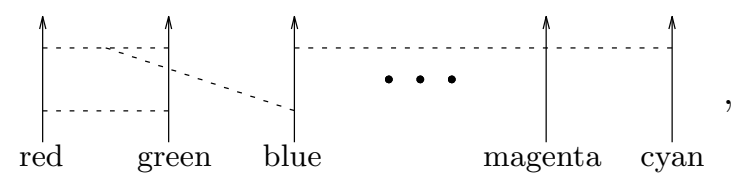

divided by the same $S T U$ relations as before. Notice that the full lines in these diagrams are colored by (all of) the colors in $\Upsilon$, and that in $\mathcal{A}^{s l}$ we consider only diagrams that do not have connected components made only of dashed arcs. As in the case of knots, the $A S$ and $I H X$ relations hold in $\mathcal{A}^{s l}$. $\mathcal{A}^{s l}$ is graded by half the number of trivalent vertices in a diagram; the diagram shown above is of degree 4 .

- $\overline{\mathcal{A}}^{s l}$ is the graded completion of $\mathcal{A}^{s l}$ and $\mathcal{A}^{s l *}$ is the graded dual of $\mathcal{A}^{s l}$. $\mathcal{W}^{s l}$ is the space of all linear functionals on $\mathcal{A}^{s l}$ which vanish on all diagrams having an arc whose endpoints are both on the same colored line and are not separated by an endpoint of any other arc. As in the case of knots, there is a naturally defined map $W_{m}^{s l}: \mathcal{F}_{m} \mathcal{V}^{s l} \rightarrow \mathcal{W}^{s l}$.

- Like $\mathcal{A}, \mathcal{A}^{\text {sl }}$ is a (co-commutative but not commutative) Hopf algebra. Furthermore, $\mathcal{A}^{\text {sl }}$ is an $\mathcal{A}$-module and $\mathcal{A}$-co-module in $k$ different ways, one way for each of the colors in $\Upsilon$. For example, if $D$ and $D^{s l}$ are diagrams representing classes in $\mathcal{A}$ and $\mathcal{A}^{s l}$ respectively, then the 'blue product' $D \times D_{\text {blue }}^{s l}$ is defined by cutting the blue line of $D^{s l}$ somewhere, and inserting $D$ into that cut. It is easy to verify that modulo $S T U, A S$ and $I H X$, the result is independent of the choice of the cutting point. The 'blue co-product' is inherited from the blue product $\times: \mathcal{V} \otimes \mathcal{V}^{s l} \rightarrow \mathcal{V}^{s l}$ defined as follows: if $\sigma$ is a string link and $V$ and $V^{s l}$ are invariants in $\mathcal{V}$ and $\mathcal{V}^{s l}$ respectively, then

$$
\left(V \underset{\text { blue }}{\times} V^{s l}\right)(\sigma)=V\left(\sigma_{\text {blue }}\right) V^{s l}(\sigma),
$$

where $\sigma_{\text {blue }}$ is the closure of the blue strand of $\sigma$ into a knot.

- An "integration" map $V^{s l}: \mathcal{W}^{s l} \rightarrow \mathcal{V}^{s l}$ can be defined as in [3]. The main difference is that the "correction" procedure now is a little more complicated - instead of multiplying by $Z(\infty)^{1-\frac{c}{2}}$, here we have to multiply using the red product by $Z(\infty)^{1-\frac{c(\mathrm{red})}{2}}$, using the green product by $Z(\infty)^{1-\frac{c(\text { green })}{2}}$, etc.

- A map $\mathcal{A}^{s l *} \rightarrow \mathcal{W}^{s l}$ can be defined using the $\mathcal{A}$-module and $\mathcal{A}$-co-module structures of $\mathcal{A}^{s l}$, in a way similar to the definition of the map $\mathcal{A}^{*} \rightarrow \mathcal{W}$.

- The space $\mathcal{B}^{\text {sl }}$ is defined as $\mathcal{B}$, only that each univalent vertex in a diagram in $\mathcal{B}^{s l}$ is colored by a color in $\Upsilon$ (color repetitions are allowed, and not all colors need to be used in any specific diagram). $\mathcal{B}^{s l}$ is isomorphic to $\mathcal{A}^{s l}$, and the isomorphism $\bar{\chi}^{s l}$ as well as its inverse $\bar{\sigma}^{s l}$ are defined as in [3], only that the summation in the definition of $\bar{\chi}^{s l}$ as well as the "uniformization" in the definition of $\bar{\sigma}^{s l}$ should be carried in each color separately.

- $\mathcal{M}^{s l}$ is defined as $\mathcal{M}$, only that the markings of a surface $M \in \mathcal{M}^{s l}$ are colored. The map $\Phi^{s l}: \mathcal{B}^{s l} \rightarrow \mathcal{M}^{s l}$ is the obvious "coloring" of the map $\Phi: \mathcal{B} \rightarrow \mathcal{M}$.

- The above spaces are all graded or filtered. Their gradations (filtrations) are the obvious generalizations of the gradations (filtrations) of the spaces in (3). 


\section{VASSILIEV HOMOTOPY STRING LINK INVARIANTS}

The purpose of this section is to describe the analogs of the maps (1) and (3) for the case of Vassiliev homotopy string link invariants, namely, for the case of Vassiliev invariants of string links which do not change when an undercrossing in which both strands are of the same color (a boring undercrossing) is changed to become an overcrossing. The map for Vassiliev homotopy string link invariants is:

$$
\begin{aligned}
& \mathcal{K}^{h s l} \underset{1-1 !}{\stackrel{V^{h s l}}{\longrightarrow}} \overline{\mathcal{A}}^{h s l} \supset \mathcal{A}^{h s l} \underset{\bar{\sigma} h s l}{\stackrel{\bar{\chi}^{h s l}}{\longrightarrow}} \mathcal{B}^{h s l} \underset{1-1 !}{\stackrel{\Phi^{h s l}}{\longrightarrow}} \mathcal{M}^{h s l} \\
& \mathcal{K}^{h s l *} \supset \mathcal{V}^{h s l} \underset{V \text { hsl }}{\stackrel{W_{m}^{h s l}}{\longrightarrow}} \mathcal{W}^{h s l}=\mathcal{A}^{h s l *} \underset{\bar{\sigma} h s l_{*}}{\stackrel{\bar{\chi}}{h s l *}} \mathcal{B}^{h s l *} \underset{\text { onto! }}{\stackrel{\Phi^{h s l *}}{\leftrightarrows}} \mathcal{M}^{h s l *}
\end{aligned}
$$

Where

- $\mathcal{K}^{h s l}$ is the space spanned by all string links, considered up to homotopy.

- The space $\mathcal{V}^{h s l}$ of Vassiliev homotopy invariants is defined in the usual way.

- $\mathcal{A}^{h s l}$ is $\mathcal{A}^{s l}$ with the further relation imposed that a diagram that has an arc both of whose ends are connected to the same full line is equal to 0. (Such arcs will be referred to as boring). $\overline{\mathcal{A}}^{\text {hsl }}$ is the graded completion of $\mathcal{A}^{\text {hsl }}$.

- $W_{m}^{h s l}$ is the restriction of $W_{m}^{s l}$ to $\mathcal{F}_{m} \mathcal{V}^{h s l}$.

- $\mathcal{W}^{h s l}$ is just the dual of $\mathcal{A}^{\text {hsl }}$. The only place in this section where homotopy invariance is used is here, in showing the rather simple fact that the image of $W_{m}^{h s l}$ is contained in $\mathcal{W}^{h s l}$.

- $\mathcal{B}^{h s l}$ is the quotient of $\mathcal{B}^{s l}$ by the subspace spanned by all non-forests, i.e. by all diagrams whose first homology is non-trivial, and by all boring diagrams - diagrams that have two (or more) univalent vertices on the same component and colored by the same color. Clearly, $\mathcal{B}^{h s l}$ is isomorphic to the space spanned by interesting (i.e. non-boring) forests.

- $\mathcal{M}^{h s l}$ is the subspace of $\mathcal{M}^{s l}$ spanned by disjoint unions of interesting disks - where an interesting disk is defined to be a disk whose markings are of distinct colors.

- All spaces above inherit gradations (or filtrations) from the corresponding spaces in (3).

The only non-obvious things to check are that the isomorphism $\mathcal{A}^{s l} \leftrightarrow \mathcal{B}^{s l}$ descends to an isomorphism $\mathcal{A}^{h s l} \leftrightarrow \mathcal{B}^{h s l}$, and that the map $\Phi^{h s l}: \mathcal{B}^{h s l} \rightarrow \mathcal{M}^{h s l}$ (whose definition is the obvious one) is one-to-one.

Theorem 1. The isomorphism $\mathcal{A}^{s l} \leftrightarrow \mathcal{B}^{s l}$ descends to an isomorphism $\mathcal{A}^{\text {hsl }} \leftrightarrow \mathcal{B}^{h s l}$.

Proof. First, let us show that relations in $\mathcal{B}^{\text {hsl }}$ are mapped into relations in $\mathcal{A}^{\text {hsl }}$ by the isomorphism $\bar{\chi}^{s l}: \mathcal{B}^{s l} \rightarrow \mathcal{A}^{s l}$. Recall that if $C$ is a diagram in $\mathcal{B}^{s l}, \bar{\chi}^{s l}(C)$ is the sum of all possible ways of arranging all the $v$-colored univalent vertices in $C$ along $v$-colored directed full lines, for all colors $v \in \Upsilon$. There are two types of (additional) relations in $\mathcal{B}^{s l}$ : 
Boring diagrams: Such diagrams are mapped by $\bar{\chi}^{s l}(C)$ to combinations of diagrams having subdiagrams that look like

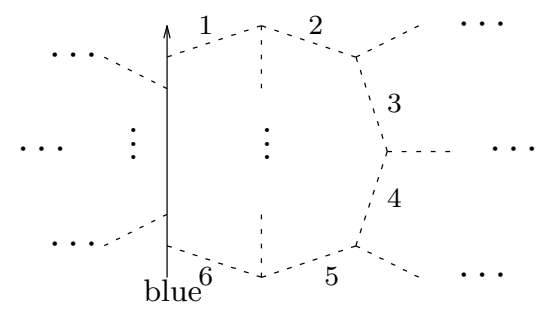

Apply the $S T U$ relation near the arc 1, regarding the displayed diagram as an $S$ diagram. In the result, $T+(-U)$, in each of the summands arc 2 is connected to the blue line. Again apply the $S T U$ relation in the same manner near arc 2 in each of the summands. The result is a larger signed sum in each of whose terms the $S T U$ relation can be applied near arc 3. Keep applying the $S T U$ relation until you reach arc 6 . In each of the resulting summands arc 6 is boring, and thus $\bar{\chi}^{s l}(C)=0$ in $\mathcal{A}^{\text {hsl }}$.

Diagrams with non-trivial first homology: Such diagrams are mapped by $\bar{\chi}^{s l}(C)$ to combinations of diagrams having subdiagrams that look like

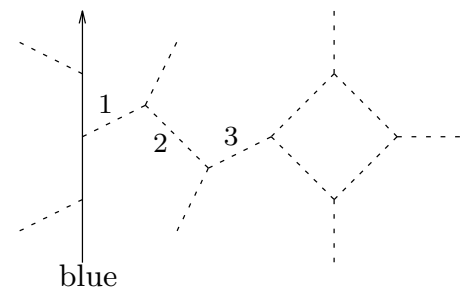

Apply the $S T U$ relation to arcs 1, 2, and 3 in sequence as before, and you get back to the previous case.

Next, let us show that relations in $\mathcal{A}^{h s l}$ are mapped into relations in $\mathcal{B}^{h s l}$ by the inverse isomorphism $\bar{\sigma}^{s l}: \mathcal{A}^{s l} \rightarrow \mathcal{B}^{s l}$. Recall that if $D$ is a diagram in $\mathcal{A}^{s l}, \bar{\sigma}^{s l}(D)$ is a certain linear combination of diagrams obtained from $D$ by first applying to $D$ some sequences of 'basic operations', and then erasing the full lines in the resulting diagrams. Recall also the two kinds of basic operations used:

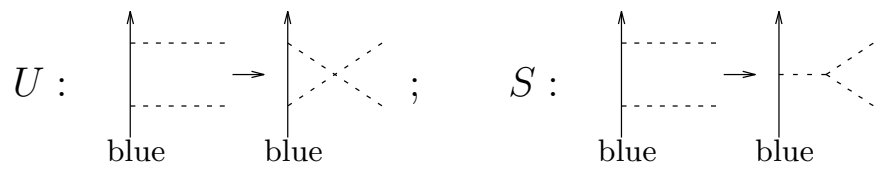

Given $D \in \mathcal{A}^{\text {sl }}$, let $D^{C C}$ be the diagram $D$ with the full lines removed. It is clear that the property " $D^{C C}$ is a relation in $\mathcal{B}^{\text {hsl" }}$ of a diagram $D$ is preserved by the operations $U$ and $S$, and that diagrams that have a boring arc have this property.

Theorem 2. The map $\Phi^{h s l}: \mathcal{B}^{h s l} \rightarrow \mathcal{M}^{\text {hsl }}$ is one-to-one.

Proof. Clearly, it is sufficient to prove the theorem for connected diagrams, and we might as well restrict our attention to diagrams whose univalent vertices are in a bijective correspondence with the colors in $\Upsilon$. Let $\mathcal{B}^{\text {res }}$ denote the corresponding subspace of $\mathcal{B}^{\text {hsl }}$. The corresponding restricted class of surfaces, $\mathcal{M}^{\text {res }}$, is the class of disks whose markings are in bijective correspondence with the colors in $\Upsilon$. Let us pick one of the colors in $\Upsilon$, say "red". With that choice, the diagrams in $\mathcal{B}^{\text {res }}$ are just binary trees with colored leaves (modulo the 
$I H X$ relation), and there is an injective map $R_{L}: \mathcal{B}^{\text {res }} \rightarrow F L(\Upsilon-$ red $)$ of $\mathcal{B}^{\text {res }}$ into the free lie algebra $F L(\Upsilon-$ red) generated by the colors in $\Upsilon$ other than red:

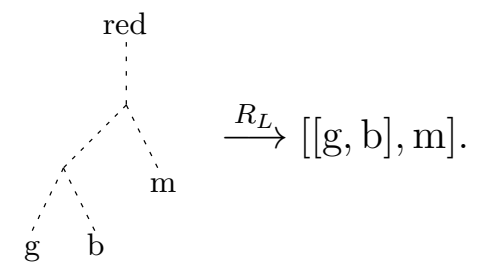

( $R_{L}$ is well defined because the $I H X$ relation maps to the Jacobi identity in $F L(\Upsilon-$ red $)$ and the $A S$ relation maps to the antisymmetry of the bracket. It is injective because the images of the $I H X$ and $A S$ relations are all the relations in $F L(\Upsilon$-red) which involve only 'interesting' Lie monomials). Similarly, there is a map $R_{A}: \mathcal{M}^{\text {res }} \rightarrow F A(\Upsilon-$ red $)$ of $\mathcal{M}^{\text {res }}$ into the free associative algebra $F A(\Upsilon$ - red) generated by $\Upsilon$ - red:

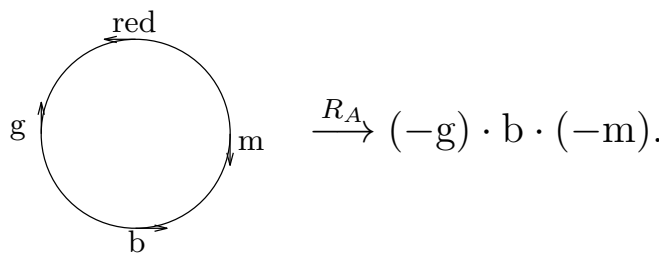

(Notice that the direction of the red marking is used to determine the order of $\mathrm{g}, \mathrm{b}$, and $\mathrm{m}$, and that we took each marking whose orientation was opposite to that of the red marking with a minus sign).

The theorem now follows from the injectivity of $R_{L}$ and of the natural map $i: F L \rightarrow F A$ of the free Lie algebra $F L$ into its universal enveloping algebra $F A$, and from the easily established commutativity of the diagram

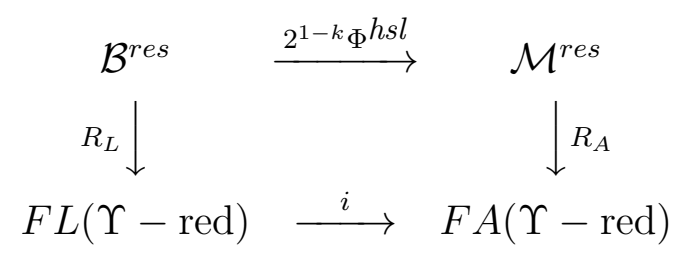

(The injectivity of $i$ is proven e.g. in [16, theorem 5.9]).

Theorem 3. $\mathcal{B}^{\text {hsl }}$ (and hence $\mathcal{A}^{\text {hsl }}$ ) is a polynomial algebra over a graded vector space $\mathcal{P}^{\text {hsl }}$ whose degree $m$ homogeneous subspace $\mathcal{G}_{m} \mathcal{P}^{\text {hsl }}$ is of dimension $\left(\begin{array}{c}k \\ m+1\end{array}\right)(m-1)$ ! for $1 \leq m<k$, and of dimension 0 otherwise.

Proof. The theorem follows from the following three assertions:

(1) $\mathcal{P}^{h s l}$ can be taken to be the subspace of $\mathcal{B}^{h s l}$ spanned by connected diagrams, i.e. by trees.

(2) A tree of degree $m$ in $\mathcal{P}^{h s l}$ is a tree with $m+1$ leafs colored by $m+1$ different colors from $\Upsilon$.

(3) The subspace $\mathcal{P}_{\Upsilon_{0}}$ of $\mathcal{P}^{h s l}$ spanned by trees whose $m+1$ leafs are colored by the colors in some fixed subset $\Upsilon_{0}$ of $\Upsilon$ is of dimension $(m-1)$ ! when $1 \leq m<k$, and of dimension 0 otherwise.

Assertion 1 follows using the same reasoning as in [3]. Assertion 2 is trivial from the definition of the grading of $\mathcal{B}^{h s l}$. Let us prove assertion 3. It is easy to check that if $m<1$ 
or $m \geq k$ then $\mathcal{G}_{m} \mathcal{P}_{\Upsilon_{0}}$ is empty. Let $1 \leq m<k$, let us fix a subset $\Upsilon_{0}$ of order $m+1$ of $\Upsilon$, and let us assume, without loss of generality, that the colors red and cyan are in $\Upsilon_{0}$. Let $P$ be the set of all diagrams of the form

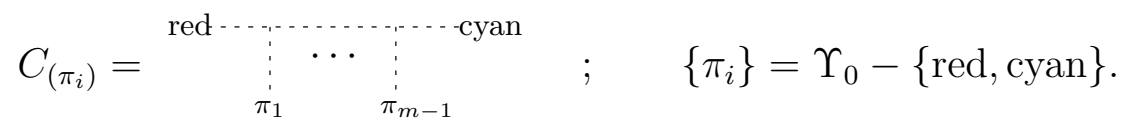

The elements of $P$ are linearly independent in $\mathcal{P}_{\Upsilon_{0}}$ : Let $\left(\pi_{i}\right)$ and $\left(\bar{\pi}_{i}\right)$ be colors so that $\left\{\bar{\pi}_{i}\right\}=\left\{\pi_{i}\right\}=\Upsilon_{0}-\{$ red,cyan $\}$, and let $M_{\left(\bar{\pi}_{i}\right)}$ be a disk whose boundary is marked by $m+1$ tangents of consistent orientations and whose colors are of the same cyclic order as (red, $\bar{\pi}_{1}, \ldots, \bar{\pi}_{m-1}$, cyan). It is easy to verify that the coefficient of $M_{\left(\bar{\pi}_{i}\right)}$ in $\Phi^{h s l}\left(C_{\left(\pi_{i}\right)}\right)$ is 2 if $\left(\pi_{i}\right)=\left(\bar{\pi}_{i}\right)$ and 0 otherwise, and this proves the linear independence of the elements of $P$.

$P$ spans $\mathcal{G}_{m} \mathcal{P}_{\Upsilon_{0}}$ : Let $C$ be a diagram representing a class in $\mathcal{G}_{m} \mathcal{P}_{\Upsilon_{0}}$, and let $l$ be the path in $C$ connecting the red univalent vertex with the cyan univalent vertex. If $l$ is of maximal length, then by $A S$ relations $C$ is equivalent up to a sign to a diagram in $P$. Otherwise, use the $I H X$ relation as in the figure below to show that $C$ is equivalent to a difference of diagrams whose $l$ 's are longer:

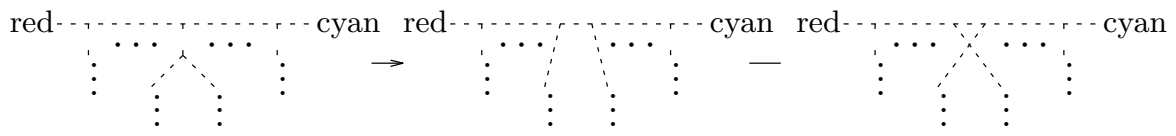

Remark 3.1. It was the computation of the dimension of $\mathcal{G}_{m} \mathcal{P}^{\text {hsl }}$ that first suggested to the author that the Milnor $\mu$ invariants are Vassiliev invariants, as will be shown in section 5 . Indeed, it is shown in $[17,18,9]$ that the number of $\mu$ invariants is given by a similar formula.

\section{VASSILIEV INVARIANTS CLASSIFY BRAIDS}

4.1. Braids. Recall (e.g. [4]) that a $k$-braid $B$ is an object that looks something like:

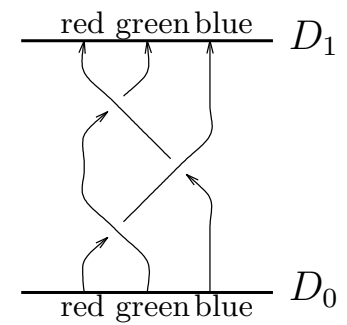

$$
\left(\begin{array}{c}
\text { More formally, } B \text { is a class in the fundamental group of } \\
\left\{\left(z_{1}, \ldots, z_{k}\right) \in D^{k}: z_{i}=z_{j} \Rightarrow i=j\right\} / S_{k}, \\
\text { where } S_{k} \text { is the group of permutations of } k \text { letters with } \\
\text { its natural action on } D^{k} \text {. We choose the class of some } \\
\text { fixed sequence }\left(\bar{z}_{1}, \ldots, \bar{z}_{k}\right) \in D^{k} \text { as a base point, and } \\
\text { associate colors in the set } \Upsilon=\left\{v_{i}\right\}=\{\text { red, }, \ldots\} \text { to each } \\
\text { of the } \bar{z}_{i} \text { 's. }
\end{array}\right)
$$

Clearly, the complement of the braid in $D \times I$ deformation retracts to the complement $D_{0}$ of the endpoints of the braid in $D \times\{0\}$, as well as to the complement $D_{1}$ of the endpoints of the braid in $D \times\{1\}$. The fundamental group $\pi_{1}\left(D_{0}\right)$ of $D_{0}$ can be identified with the free group $F(\Upsilon)$ generated by the set $\Upsilon$. Identifying $\pi_{1}\left(D_{1}\right)$ in the same way, we get the following isomorphisms:

$$
F(\Upsilon) \simeq \pi_{1}\left(D_{0}\right) \simeq \pi_{1}(D \times I-B) \simeq \pi_{1}\left(D_{1}\right) \simeq F(\Upsilon)
$$

Composing these isomorphisms from left to right, we see that to every pure braid $B$ corresponds an automorphism $\xi_{B}$ of the free group $F(\Upsilon)$. Artin's theorem (see e.g. [4, pp. 25]) says that, in fact, the braid $B$ can be reconstructed from the automorphism $\xi_{B}$. 
Let $P(\Upsilon)$ be the ring of formal power series with rational coefficients in the non-commutative variables $\left\{\bar{v}_{i}\right\}$. Recall that the Magnus expansion (see [16]) is the injective ring homomorphism $\zeta: F(\Upsilon) \rightarrow P(\Upsilon)$ defined by $^{2} \zeta\left(v_{i}\right)=\exp \left(\bar{v}_{i} / 2\right)$ and by $\zeta\left(v_{i}^{-1}\right)=\exp \left(-\bar{v}_{i} / 2\right)$. Let $\Pi_{m}: P(\Upsilon) \rightarrow \mathcal{G}_{m} P(\Upsilon)$ be the projection to the degree $m$ subspace $\mathcal{G}_{m} P(\Upsilon)$ of $P(\Upsilon)$. The following two propositions prove the assertion in the title of this section — that Vassiliev invariants are sufficient to separate braids:

Proposition 4.1. A braid $B$ is determined by the elements $\left(\zeta\left(\xi_{B}(v)\right)\right)_{v \in \Upsilon}$ of $P(\Upsilon)$.

Proof. Follows immediately from Artin's theorem and the injectivity of $\zeta$.

Proposition 4.2. For any $v \in \Upsilon, B \mapsto \Pi_{m} \zeta\left(\xi_{B}(v)\right)$ is a Vassiliev invariant of type $m-1$ (using the obvious definition of $\mathcal{G}_{m} P(\Upsilon)$-valued Vassiliev invariants of braids).

Remark 4.3. Kohno in [13] used deep results from algebraic geometry to prove that a certain class of invariants, constructed via an analog of the Knizhnik-Zamolodchikov connection, is strong enough to classify braids. Invariants coming from the Knizhnik-Zamolodchikov connection are always of finite type, and so his result implies ours. However, the proof presented here is considerably simpler and its generalizations in the case of string links are essentially obvious. Furthermore, it appears likely that with relatively little additional effort enough combinatorial information can be deduced from our line of thought to answer problems like problem 6.2.

4.1.1. The Wirtinger presentation. To prove proposition 4.2, we first need to understand $\xi_{B}$ better, or, in other words, to understand $\pi_{1}(D \times I-B)$ better. Let us recall the Wirtinger presentation (see e.g. [20]) of the fundamental group of the complement $K^{c}$ of any "knotty" object $K$ (a knot, link, braid, string link, ...) in $\mathbb{R}^{3}$ (or a contractible subset thereof). According to the Wirtinger presentation, $\pi_{1}\left(K^{c}\right)$ has one generator for each arc segment $l$ in a planar projection of $K$, denoted by an arrow $\gamma_{l}$ crossing under $l$ (to be thought of as a loop beginning at a base point above the plane, reaching the tail of $\gamma_{l}$ by a straight line, following $\gamma_{l}$, and returning on a straight line to the basepoint). It is convenient to choose all arrows along any single strand of $K$ to have consistent orientations, and than the relations among the $\gamma_{l}$ 's in $\pi_{1}\left(K^{c}\right)$ can be read from the crossings of $K$ as follows:

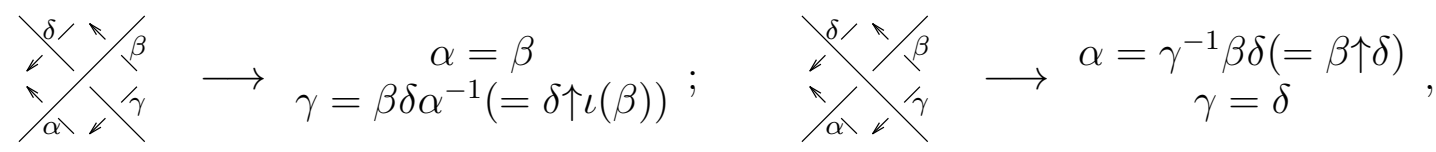

where in a group $\alpha \uparrow \beta \stackrel{\text { def }}{=} \alpha^{\beta}=\beta^{-1} \alpha \beta$ and $\iota(\alpha) \stackrel{\text { def }}{=} \alpha^{-1}$.

For example, the fundamental group of the complement of the trefoil knot in $\mathbb{R}^{3}$ is computed as follows:

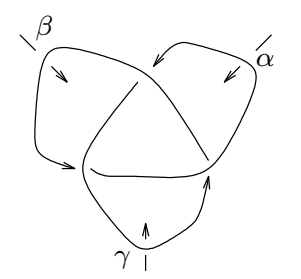

$$
\longrightarrow\langle\alpha, \beta, \gamma \mid \alpha=\beta \uparrow \gamma, \beta=\gamma \uparrow \alpha, \gamma=\alpha \uparrow \beta\rangle .
$$

\footnotetext{
${ }^{2}$ Our definition of $\zeta$ is different than the standard one, which is $\zeta\left(v_{i}\right)=1+\bar{v}_{i} ; \zeta\left(v_{i}^{-1}\right)=1-\bar{v}_{i}+\bar{v}_{i}^{2}-\ldots$. We feel that in a subject neighboring the subject of quantum groups $[6,21]$, ours would be the better definition in the long run.
} 
4.1.2. Understanding $\xi_{B}(v)$. It is now rather simple to understand how $\xi_{B}$ works. To compute $\xi_{B}(v)$, start from the generator of $\pi_{1}(D \times I-B)$ that corresponds to $v$ - an arrow passing under the very bottom of the strand whose lower end is the point of color $v$. Then 'slide' this generator upward, while occasionally conjugating it by some other generator (or its inverse), as dictated by the relations (7). Then slide the conjugators upward using the same procedure, and then the conjugators of the conjugators, .... As the right hand sides of the relations 7 only involve generators that are higher up along the braid than the left hand sides, this process will terminate. More formally, we can describe this process as follows:

- Write an arrow, marked by a greek letter, under each arc segment in a planar projection of $B$. For the arc segments on the first strand of the braid use the letters $\alpha_{0}, \ldots, \alpha_{n_{\alpha}}=\alpha$, for the second strand use $\beta_{0}, \ldots, \beta_{n_{\beta}}=\beta$, etc.

- Start from the generator $v$, say $\beta_{0}$ according to the new marking, and repeatedly apply (7) replacing left-hand-sides by right-hand-sides until you get an expression for $\beta_{0}$ in terms of $\alpha, \beta, \ldots$. This expression is the sought after $\xi_{B}(v)$.

For example, consider $v=\beta_{0}$ in the case of the braid given in (5):

$$
\beta_{0} \rightarrow \beta_{1} \rightarrow \beta_{2} \uparrow \gamma_{2}=\beta \uparrow \gamma
$$

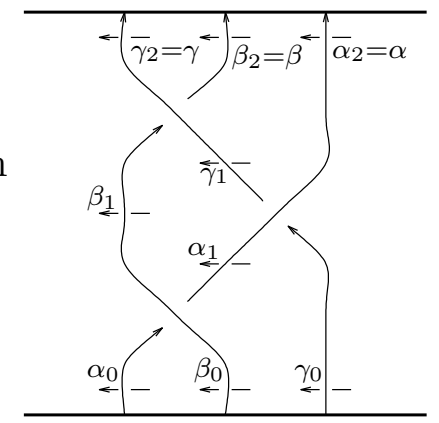

4.1.3. Idea of the proof. The idea of the proof of proposition 4.2 is simple: looking at (7) we see that a double point corresponds to the difference between conjugating and not conjugating. Such a difference between, say, $\alpha \uparrow \beta$ and $\alpha$ vanishes if either $\alpha=e$ or $\beta=e$ where $e$ is the identity element of a group, thus the Magnus expansion of such a difference is divisible by both $\alpha$ and $\beta$ and hence it cannot have terms of degree less than 2 . (Indeed, the lowest degree term in $\zeta(\alpha \uparrow \beta)-\zeta(\alpha)$ is $[\bar{\alpha}, \bar{\beta}]$, which is of degree 2$)$. Having more double points in $B$ should then mean that the lowest degree term in $\xi_{B}(v)$ is of a higher and higher degree. The rather messy details of this simple idea are shown in the following section.

4.2. Braids with double points. Fixing a color $v$, it is clear that one can use (2) to extend $\xi_{B}(v)$ to be defined on braids $B$ that are allowed to have double points, provided that we allow $\xi_{B}(v)$ to take values in the group ring $\mathbb{Z F}(\Upsilon)$ of the free group $F(\Upsilon)$. Being a little more specific, say that $B$ has exactly $m$ double points and mark them by the integers $1, \ldots, m$. Define a new (and not too interesting) operation $\downarrow: F(\Upsilon) \times F(\Upsilon) \rightarrow F(\Upsilon)$ by $\alpha \downarrow \beta=\alpha$. Introduce new "meta-operations" $C_{i}, 1 \leq i \leq m$ on $F(\Upsilon)$ to be assigned a meaning shortly, and add two new rules to the rules in (7):

$$
\begin{gathered}
\alpha \rightarrow \beta\left(C_{i}\right)^{o p} \delta \\
\gamma \rightarrow \delta C_{i} \iota(\beta)
\end{gathered} . \quad\left(\begin{array}{c}
\left(C_{i}\right)^{o p} \text { will also be } \\
\text { assigned a } \\
\text { meaning shortly }
\end{array}\right)
$$


Claim 4.4. Let $\bar{\xi}_{B}(v)$ denote the result of the procedure (8) supplemented by the additional rule (9) applied to the braid $B$ beginning from the generator $v$. Then

$$
\xi_{B}(v)=\sum_{\left(C_{i}\right) \in\{\uparrow, \downarrow\}^{m}}(-1)^{\left|\left\{i: C_{i}=\downarrow\right\}\right|} \cdot \bar{\xi}_{B}(v) . \quad\left(\begin{array}{c}
\text { where } \\
(\uparrow)^{o p}:=\downarrow \text { and } \\
(\downarrow)^{o p}:=\uparrow
\end{array}\right)
$$

(Notice that in the above sum whenever $\bar{\xi}_{B}(v)$ is evaluated the meta-operations $C_{i}$ (as well as their opposites $\left(C_{i}\right)^{\text {op }}$ ) are assigned a definite meaning, which is either $\uparrow$ or $\left.\downarrow\right)$.

Let us assume that in the formal expression $\bar{\xi}_{B}(v)$ the meta-operation $C_{i}$ appears $n_{i}$ times. Introduce new meta-operations $C_{i}^{j}, 1 \leq i \leq m ; 1 \leq j \leq n_{i}$, and let $T$ be the formal expression obtained from $\bar{\xi}_{B}(v)$ by replacing the $j$ th occurrence of $C_{i}$ in $\bar{\xi}_{B}(v)$ by $C_{i}^{j}$, for all $i$ and $j$ in the range $1 \leq i \leq m ; 1 \leq j \leq n_{i}$. Let $H$ by the hypercube $\prod_{i}\left[0, n_{i}\right)$ in $\mathbb{R}^{m}$, and let $\bar{H}$ be its closure ${ }^{3}$. For an integer point $p=\left(p_{i}\right) \in \mathbb{Z}^{m} \cap \bar{H}$ set

$$
T_{p}=\left(T \text { with all } C_{i}^{j} \text { s replaced by }\left\{\begin{array}{ll}
\uparrow & \text { if } j \leq p_{i}, \\
\downarrow & \text { otherwise }
\end{array}\right)\right.
$$

and for $p \in \mathbb{Z}^{m} \cap H$ let

$$
T(p)=\sum_{\epsilon=\left(\epsilon_{i}\right) \in\{0,1\}^{m}}(-1)^{\sum \epsilon_{i}} \cdot T_{p+\epsilon} .
$$

Clearly, the previous claim just says that $\xi_{B}(v)$ is equal to the alternating sum of the values of $T_{p}$ on the corners of $\bar{H}$. With this in mind, the following lemma is just an $m$-dimensional generalization of the notion of "a telescopic sum":

Lemma 4.5. $\xi_{B}(v)=\sum_{p \in \mathbb{Z}^{m} \cap H} T(p)$.

4.2.1. Proof of proposition 4.2. Using the above lemma, we see that it is sufficient to prove that $\Pi_{m} \zeta T(p)=0$ for each $p \in \mathbb{Z}^{m} \cap H$. Fix such a $p$ once and for all. Extend the operations $\uparrow$ and $\downarrow$ to bilinear ${ }^{4}$ operations on $\mathbb{Z} \mathbb{F}(\Upsilon)$, and the operation $\iota$ to a linear ${ }^{5}$ operation on $\mathbb{Z} \mathbb{F}(\Upsilon)$. Define $*: \mathbb{Z} F(\Upsilon) \otimes \mathbb{Z} \mathbb{F}(\Upsilon) \rightarrow \mathbb{Z} \mathbb{F}(\Upsilon)$ to be the bilinear extension of the operation $(\alpha, \beta) \mapsto \alpha \uparrow \beta-\alpha \downarrow \beta$ defined on $F(\Upsilon) \times F(\Upsilon)$, and set $(*)^{o p}=(-*)$. Let

$$
T^{*}(p)=\left(T \text { with all } C_{i}^{j} \text { s s replaced by }\left\{\begin{array}{ll}
\uparrow & \text { if } j \leq p_{i}, \\
* & \text { if } j=p_{i}+1, \\
\downarrow & \text { otherwise }
\end{array}\right)\right. \text {. }
$$

Lemma 4.6. $T^{*}(p)=T(p)$.

For a subset $A \subset \Upsilon$ of $\Upsilon$, let $\Pi_{A}: F(\Upsilon) \rightarrow F(\Upsilon-A)$ be the natural projection map that maps all the members of $A$ to the identity element $e$ of $F(\Upsilon-A)$, and use the same symbol $\Pi_{A}$ to denote the linear extension of $\Pi_{A}$ to a projection $\Pi_{A}: \mathbb{Z} \mathbb{F}(\Upsilon) \rightarrow \mathbb{Z} \mathbb{F}(\Upsilon-A)$.

\footnotetext{
${ }^{3}$ The rest of this section doesn't make much sense if $n_{i}=0$ for some $i$. In that case, however, $\xi_{B}(v)=0$ and there is nothing to prove.

${ }^{4}$ This is a rather non-standard construction; we define $\alpha \uparrow(\beta+\gamma)=\alpha \uparrow \beta+\alpha \uparrow \gamma \neq(\beta+\gamma)^{-1} \alpha(\beta+\gamma)$. (The latter possibility does not even make sense as $\beta+\gamma$ is, in general, not invertible).

${ }^{5}$ So $\iota(7 \alpha)=7 \iota(\alpha) \neq(7 \alpha)^{-1}$.
} 
Lemma 4.7. Let $E$ be any formula made of the operations $\iota, \uparrow, \downarrow$, and $*$ and of the generators $\Upsilon$ of $\mathbb{Z F}(\Upsilon)$ which involves each such generator at most once. Assume that the number $m$ of times that the operation $*$ appears in $E$ is positive. Then there exist $m+1$ disjoint non-empty subsets $\left\{A_{i}\right\}_{i=1}^{m+1}$ of $\Upsilon$ for which $\Pi_{A_{i}}(E)=0$.

Assuming the lemma, the proof of the vanishing of $\Pi_{m} \zeta T(p)=\Pi_{m} \zeta T^{*}(p)$ is short. First notice that setting $E=T^{*}(p)$, the operation $*$ appears precisely $m$ times in $E$. If $E$ involves each generator of $F(\Upsilon)$ at most once, the lemma shows that there are $m+1$ projections $\bar{\Pi}_{A_{i}}: P(\Upsilon) \rightarrow P(\Upsilon-A)$ (whose obvious definitions are left as an exercise for the reader) for which $\bar{\Pi}_{A_{i}}\left(T^{*}(p)\right)=0$. This implies that each monomial in $\zeta\left(T^{*}(p)\right)$ contains at least one variable from each of the $A_{i}$ 's, and is therefore at least of degree $m+1$. This shows that $\Pi_{m} \zeta\left(T^{*}(p)\right)=0$. Having repetitions among the generators appearing in $E$ is just the same as considering a 'lifting' $\tilde{E}$ of $E$ to a formula in which there are no repeating variables, and then imposing some (equality) relations among the variables appearing in $\tilde{E}$. But if something $\left(\Pi_{m} \zeta(\tilde{E})\right)$ vanishes, it vanishes no matter how many further relations are imposed.

4.2.2. Proof of lemma 4.\%. The proof is by induction on the structure of $E$.

Case 1: $E$ is a generator of $F(\Upsilon)$. In this case there is nothing to prove.

Case 2: $E=\iota\left(E^{1}\right)$. If $\left\{A_{i}^{1}\right\}$ are the subsets of $\Upsilon$ corresponding to $E^{1}$ by the induction hypothesis, simply set $A_{i}=A_{i}^{1}$.

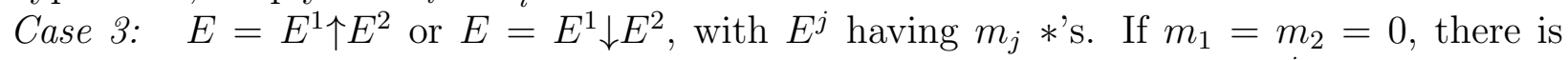
nothing to prove. Otherwise let $\left\{A_{i}\right\}=\left\{A_{i}^{1}\right\} \cup\left\{A_{i}^{2}\right\}$ (where if $m_{j}=0,\left\{A_{i}^{j}\right\}$ is understood to be the empty collection), and use the linearity of $\uparrow$ and $\downarrow$. Observe that if both $m_{j}$ 's are positive, we have $m_{1}+m_{2}+2$ sets $A_{i}$, which is one more than the required number $m+1=m_{1}+m_{2}+1$.

Case 4: $E=E^{1} * E^{2}$, with $E^{j}$ having $m_{j} *$ 's. If $m_{1}=m_{2}=0$, take $A_{i}=$ all generators appearing in $\left.E^{i}\right\}$, and use the facts that $e \uparrow a-e \downarrow a=e^{a}-e=0$ and $a \uparrow e-a \downarrow e=a^{e}-a=0$ for any $a \in F(\Upsilon)$. If both $m_{j}$ 's are positive, use the same construction and the same observation as in the previous case. If $m_{1}>0$ and $m_{2}=0$, set $A_{i}=A_{i}^{1}$ for $i \leq m$ and $A_{m+1}=\left\{\right.$ all generators appearing in $\left.E^{2}\right\}$, and if $m_{1}=0$ and $m_{2}>0$, set $A_{i}=A_{i}^{2}$ for $i \leq m$ and $A_{m+1}=\left\{\right.$ all generators appearing in $\left.E^{1}\right\}$.

\section{On THE MiLnOR INVARIANTS}

5.1. Vassiliev invariants classify string links up to homotopy. Let us try to naively imitate the procedure of (8) in the case of a string link $\sigma$ :

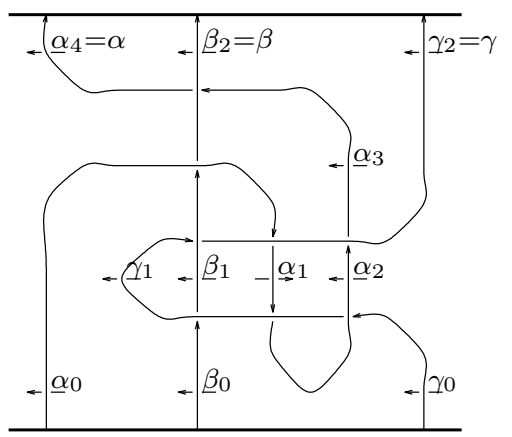

$$
\begin{aligned}
& \beta_{0} \rightarrow \beta_{1} \uparrow \gamma_{1} \\
& \beta_{1} \rightarrow \beta \uparrow \iota\left(\alpha_{0}\right) \\
& \alpha_{0} \rightarrow \alpha_{1} \uparrow \gamma \\
& \alpha_{1} \rightarrow \alpha_{2} \uparrow \iota\left(\gamma_{1}\right) \\
& \gamma_{1} \rightarrow \gamma \uparrow \beta_{1} \\
& \beta_{1} \rightarrow ?
\end{aligned}
$$

We find ourselves stuck in an infinite loop. There are several ways out, though. The simplest of these is to declare that all the conjugates of $\beta_{0}$ commute. This done, notice that in 
computing $\xi_{\sigma}\left(\beta_{0}\right)$ all the intermediate results are conjugates of $\beta_{0}$ and therefore conjugating such an intermediate result by a conjugate of $\beta_{0}$, say $\beta_{1}$, is superfluous and the above infinite loop can be avoided. This simple-minded argument can be enhanced to a complete proof of the following theorem, first proven by Milnor [17] (for a proof somewhat different from the one hinted here, see [9]):

Definition 5.1. (Habegger-Lin [9]) If $G$ is a group normally generated by $x_{1}, \ldots, x_{k}$, set

$$
R G=G /\left\langle\left[x_{i} \uparrow g_{1}, x_{i} \uparrow g_{2}\right]: g_{1,2} \in G\right\rangle .
$$

Theorem 4. The inclusions $D_{0} \hookrightarrow D \times I-\sigma \hookleftarrow D_{1}$ (see (6)) induce isomorphisms

$$
R F(\Upsilon) \simeq R \pi_{1}\left(D_{0}\right) \simeq R \pi_{1}(D \times I-\sigma) \simeq R \pi_{1}\left(D_{1}\right) \simeq R F(\Upsilon)
$$

Let $R \xi_{\sigma}$ be the left to right composition of these isomorphism.

Proposition 5.2. The Magnus expansion $\zeta: F(\Upsilon) \rightarrow P(\Upsilon)$ descends to an injection $R \zeta$ : $R F(\Upsilon) \rightarrow R P(\Upsilon)$, where $R P(\Upsilon)$ is obtained from $R P(\Upsilon)$ by setting all boring monomials, monomials in which any generator appears more than once, to be equal to 0.

Theorem 5. $\sigma \mapsto \Pi_{m} R \zeta\left(R \xi_{\sigma}(v)\right)$ is a Vassiliev invariant of type $m-1$ for every $v \in \Upsilon$.

Proof. Simply observe that the same procedure (8) for computing $\xi_{\sigma}(v)$ works here just as well, provided that when working your way up $\sigma$, you ignore every conjugator that corresponds to a strand of $\sigma$ which is being visited for the second time in the current branch of the computation tree. The result is a formula for $R \xi_{\sigma}(v)$ of the same type as in the proof of proposition 4.2, and exactly the same proof as there works here as well.

It is rather clear that $R \xi_{\sigma}$ is, in fact, a homotopy invariant of $\sigma$ : Say there is an overcrossing in $\sigma$ in which only one strand of $\sigma$, say the one marked by $\gamma_{i}$, is involved. Notice that all the $\gamma_{i}$ 's are conjugate to each other, and therefore they all commute in $R \pi_{1}(D \times I-\sigma)$. Thus the rules corresponding to this overcrossing are both trivial, and equal to the rules that would apply had it been an undercrossing:

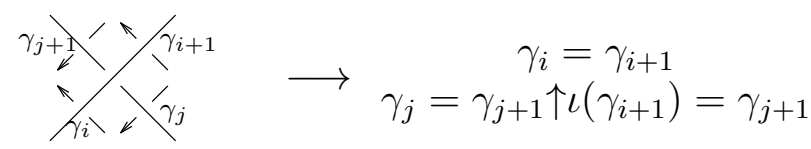

Theorem 6. (Habegger-Lin [9]) R $\xi_{\sigma}$ determines $\sigma$ up to homotopy.

Thus we see that Vassiliev invariants classify string links up to homotopy.

5.2. The Milnor $\mu$ invariants. The invariants considered in the previous section, $\sigma \mapsto$ $\Pi_{m} R \zeta\left(R \xi_{\sigma}(v)\right)$, are not quite the Milnor $\mu$ invariants of string links, but they are close relatives. It is easy to see from, say, (8) that for any $v, R \xi_{\sigma}(v)$ is a conjugate of $v$. The $\mu$ invariants (without repeating indices) are just the coefficients of the Magnus expansion of a particular member $\mu_{v}$ of $R F(\Upsilon)$ for which $R \xi_{\sigma}(v)=v \uparrow \mu_{v}$ : the image via $R \pi_{1}(D \times I-$ $\sigma) \rightarrow R F(\Upsilon)$ of any parallel of the strand $v$. This image can be computed using the same techniques ((8) and (9)) as before, and so our proof also shows that those Milnor $\mu$ invariants are Vassiliev invariants.

Remark 5.3. Notice that the above result, together with the results of section 3 , show that the (no-repeating-indices) Milnor $\mu$ invariants come from the same single uniform construction as the HOMFLY and Kauffman polynomials. 
5.3. Some more Milnor $\mu$ invariants. There is a second way, also discovered by Milnor, to break out of the infinite loop of (10). Define the depth of a term $v$ appearing in a formula $E$ made of constants and the operations $\uparrow, \downarrow$, and $\iota$, to be 1 plus the number of $\uparrow \mathrm{s}$ and $\downarrow \mathrm{s}$ in $E$ whose right-hand scope ${ }^{6}$ includes $v$. Notice that the problem in (10) occurs at higher and higher depths, and so if high depths are declared irrelevant, the problem is removed. More precisely, for a group $G$ recall that the $q$ th term $G_{q}$ in the lower central series of $G$ is defined recursively by

$$
G_{1}=G ; \quad G_{q+1}=\left[G, G_{q}\right], \quad\left(\begin{array}{c}
\text { where }[g, h]:=h^{-1} g h g^{-1}=(g \uparrow h) g^{-1} \\
\text { is an equivalent but non-standard } \\
\text { definition for the commutator. }
\end{array}\right)
$$

and that the $q$ th nilpotent quotient $R_{q} G$ of $G$ is $G / G_{q}$.

The following proposition is well known, but I could not find it formulated in this form in the literature. For completeness, I've included a brief proof.

Proposition 5.4. Let $E$ be a formula made of constants and the operations $\uparrow$, $\downarrow$, and $\iota$. When $E$ is evaluated in $R_{q} G$, the result is independent of the terms in $E$ whose depth is $q$ or higher.

Proof. Set $\Uparrow(\alpha)=\alpha, \Uparrow\left(\alpha_{1}, \ldots, \alpha_{q}\right)=\alpha_{1} \uparrow \Uparrow\left(\alpha_{2}, \ldots, \alpha_{q}\right)$, and

$$
Q\left(\alpha_{1}, \ldots, \alpha_{q}\right)=\Uparrow\left(\alpha_{1}, \ldots, \alpha_{q}\right) \Uparrow\left(\alpha_{1}, \ldots, \alpha_{q-1}\right)^{-1} .
$$

To prove the proposition, it is sufficient to show that $Q\left(\alpha_{1}, \ldots, \alpha_{q}\right) \in G_{q}$. The first two cases are:

$$
\begin{gathered}
Q(\alpha, \beta)=(\alpha \uparrow \beta) \alpha^{-1}=[\alpha, \beta], \\
Q(\alpha, \beta, \gamma)=(\alpha \uparrow(\beta \uparrow \gamma))(\alpha \uparrow \beta)^{-1}=[\alpha,[\beta, \gamma]] \uparrow \beta=[\alpha, Q(\beta, \gamma)] \uparrow \uparrow(\beta) .
\end{gathered}
$$

The general case follows using induction and

$$
Q\left(\alpha_{1}, \ldots, \alpha_{q}\right)=\left[\alpha_{1}, Q\left(\alpha_{2}, \ldots, \alpha_{q}\right)\right] \uparrow \Uparrow\left(\alpha_{2}, \ldots, \alpha_{q-1}\right) .
$$

From this point, the discussion proceeds as in sections 5.1 and 5.2. The above proposition is used to show that when restricting to $R_{q} \pi_{1}(D \times I-\sigma)$, the procedure (8) may be stopped whenever the depth exceeds $q$, and therefore it always terminates. This allows one to define inverses to the natural maps $R_{q} \pi_{1}\left(D_{0}\right) \rightarrow R_{q} \pi_{1}(D \times I-\sigma) \leftarrow R_{q} \pi_{1}\left(D_{1}\right)$ and hence get a chain of isomorphisms (for a different approach see e.g. [9]):

$$
R_{q} F(\Upsilon) \simeq R_{q} \pi_{1}\left(D_{0}\right) \simeq R_{q} \pi_{1}(D \times I-\sigma) \simeq R_{q} \pi_{1}\left(D_{1}\right) \simeq R_{q} F(\Upsilon)
$$

Composing the resulting automorphism $R_{q} \xi_{\sigma}$ of $R_{q} F(\Upsilon)$ with the reduced Magnus expansion $R_{q} \zeta$, we get $R_{q} P(\Upsilon)$-valued invariants, where $R_{q} P(\Upsilon)$ is obtained from $P(\Upsilon)$ by setting all monomials of degrees $\geq q$ in $P(\Upsilon)$ to be equal to 0 . The same proof as before shows that the resulting invariants $\sigma \mapsto \Pi_{m} R_{q} \zeta R_{q} \xi_{\sigma}(v)$ are of finite type. The relation between these invariants and the Milnor $\mu$ invariants (with arbitrary indices) of string links is the same as the relation between $\sigma \mapsto R \zeta R \xi_{\sigma}(v)$ and the no-repeating-indices $\mu$ invariants, and again, there is no difficulty in showing that the newer $\mu$ invariants are Vassiliev invariants.

Remark 5.5. Lin [15] has proven in a different way that the Milnor $\mu$ invariants are Vassiliev invariants.

\footnotetext{
${ }^{6}$ So, for example, in $(\alpha \downarrow \beta) \uparrow(\gamma \uparrow(\delta \uparrow \epsilon))$, the depths of $\alpha, \beta, \gamma, \delta, \epsilon$ are $1,2,2,3,4$ respectively.
} 


\section{OdDS AND ENDS}

\subsection{Some questions.}

Problem 6.1. Does the image of $\mathcal{M}^{s l *}$ in $\mathcal{K}^{s l *}$ correspond to some class of "polynomial invariants' of string links the way the image of $\mathcal{M}^{*}$ in $\mathcal{K}^{*}$ correspond to the HOMFLY and Kauffman polynomials? Asked about $\mathcal{M}^{h s l}$ and $\mathcal{K}^{h s l}$, this problem is similar to asking whether the no-repeating-indices Milnor $\mu$ invariants all come from link polynomials.

Problem 6.2. Link the two parts of this paper. In other words, find how to express the Vassiliev invariant corresponding to an interesting disk as in (4) in terms of the invariants of section 5.1.

Problem 6.3. $\mathcal{B}^{\text {hsl }}$ is the intersection of two subspaces of $\mathcal{B}^{s l}$ - the subspace $\mathcal{B}^{\text {int }}$ of interesting diagrams and the subspace $\mathcal{B}^{\text {for }}$ of forests. This intersection was shown here to correspond to homotopy theory of string links. Can anything be said about the string links invariants corresponding to either $\mathcal{B}^{\text {int* }}$ or $\mathcal{B}^{\text {for*? }}$

Problem 6.4. A possibly related problem: On the maps (3) and (4), where are the invariants $\sigma \mapsto \Pi_{m} R_{q} \zeta R_{q} \xi_{\sigma}(v)$ ? Do they correspond to linear functionals on $\mathcal{B}^{\text {for }}$ ?

Problem 6.5. $\mathcal{A}^{\text {sl }}$ is a co-commutative but not commutative Hopf algebra. By the structure theory of such algebras [19], $\mathcal{A}^{s l}$ is the universal enveloping algebra of the Lie algebra $\mathcal{P}^{s l}$ of primitives of $\mathcal{A}^{s l}$. What is $\mathcal{P}^{s l}$ ? Notice that the structure of the analogues Lie algebra in the case of braids is understood, as in [7, pp. 847].

Problem 6.6. Develop a similar theory for links, rather than string links. The links analog of $\mathcal{A}^{s l}$ is clear - simply replace all the directed lines in the diagrams making $\mathcal{A}^{s l}$ by circles. But unlike in the case of knots, this is not a trivial operation, and, in particular, it is not clear what the analog of $\mathcal{B}^{s l}$ should now be.

6.2. Acknowledgement. I am indebted to N. Bergeron, C. Day, M. Kontsevich, J. Levine and X-S. Lin for teaching me about homotopy link invariants and in particular to M. Kontsevich and X-S. Lin for pointing out some mistakes in my initial understanding of the subject and in earlier versions of this paper. I wish to thank the anonymous referee for his comments.

\section{REFERENCES}

[1] D. Bar-Natan, Weights of Feynman diagrams and the Vassiliev knot invariants, February 1991, Preprint.

[2] _ Perturbative aspects of the Chern-Simons topological quantum field theory, Ph.D. thesis, Princeton Univ., June 1991, Dep. of Mathematics.

[3] _ On the Vassiliev knot invariants, Topology, to appear.

[4] J. S. Birman, Braids, links, and mapping class groups, Princeton Univ. Press, Princeton, 1975.

[5] and X-S. Lin, Knot polynomials and Vassiliev's invariants, Invent. Math. 111 (1993) 225-270.

[6] V. G. Drinfel'd, Quantum groups, Proc. Int. Cong. Math., Berkeley 1986.

$[7] \_$_ On quasitriangular Quasi-Hopf algebras and a group closely connected with Gal( $(\overline{\mathbb{Q}} / \mathbb{Q})$, Leningrad Math. J. 2 (1991) 829-860.

[8] M. Gusarov, A new form of the Conway-Jones polynomial of oriented links, Nauchn. Sem. Len. Otdel. Mat. Inst. Steklov 193 (1991), 4-9.

[9] N. Habegger and X-S. Lin, The Classification of links up to link-homotopy, J. Amer. Math. Soc. 3 (1990) 389-419. 
[10] J. Hoste, A. Ocneanu, K. Millett, P. Freyd, W. B. R. Lickorish and D. Yetter, A new polynomial invariant of knots and links, Bull. Amer. Math. Soc. 12 (1985) 239-246.

[11] L. H. Kauffman, On knots, Princeton Univ. Press, Princeton, 1987.

[12] _ An invariant of regular isotopy, Trans. Amer. Math. Soc. 312 (1990) 417-471.

[13] T. Kohno, Monodromy representations of braid groups and Yang-Baxter equations, Ann. Inst. Fourier 37 (1987) 139-160.

[14] X-S. Lin, Vertex models, quantum groups and Vassiliev's knot invariants, Columbia Univ. preprint, 1991.

[15] _ Milnor link invariants are all of finite type, Columbia Univ. preprint, 1992.

[16] W. Magnus, A. Karrass and D. Solitar, Combinatorial group theory: presentations of groups in terms of generators and relations, Wiley, New York, 1966.

[17] J. W. Milnor, Link groups, Annals of Math. 59 (1954) 177-195.

[18] _ Isotopy of links, Algebraic geometry and topology, A symposium in honor of S. Lefchetz, Princeton Univ. Press, Princeton 1957.

[19] _ and J. Moore, On the structure of Hopf algebras, Annals of Math. 81 (1965) 211-264.

[20] D. Rolfsen, Knots and Links, Publish or Perish, Mathematics Lecture Series 7, Wilmington 1976.

[21] V. G. Turaev, The Yang-Baxter equation and invariants of links, Invent. Math. 92 (1988) 527-553.

[22] V. A. Vassiliev, Cohomology of knot spaces, Theory of Singularities and its Applications (Providence) (V. I. Arnold, ed.), Amer. Math. Soc., Providence, 1990.

[23] V. A. Vassiliev, Complements of discriminants of smooth maps: topology and applications, Trans. of Math. Mono. 98, Amer. Math. Soc., Providence, 1992.

Department of Mathematics, Harvard University, Cambridge, MA 02138

Current address: Institute of Mathematics, The Hebrew University, Giv'at-Ram, Jerusalem 91904, Israel E-mail address: dror@math.huji.ac.il 